\title{
A CLASS OF FINITE GROUPS ADMITTING CERTAIN SHARP CHARACTERS II
}

\author{
TAKASHI MATSUHISA AND HIROYOSHI YAMAKI
}

(Communicated by Warren J. Wong)

Dedicated to Professor Tuyoshi Oyama on his sixtieth birthday

\begin{abstract}
A triple $(G, \chi, l)$ of a finite group $G$ with a faithful character $\chi$ and a character value $l$ is called sharp if

$$
\frac{a(l)}{|G|} \prod_{k \in L \backslash\{l\}}(l-k)
$$

is a unit in algebraic integers (where $l \in L$, the set of character values of $\chi$, and $a(l)$ is the number of $x \in G$ with $\chi(x)=l$ ), which generalizes the notion of sharply multiply transitive permutation groups. In this note, we shall determine sharp triples admitting the character values $L^{\sharp}=\operatorname{Im} \chi \backslash\{\chi(1)\}$ as follows: (i) $L^{\sharp}$ consists of roots of unity together with $0 \in \mathbf{Z}$, and (ii) $L^{\sharp}=\left\{0, l_{1}, l_{2}, \ldots, l_{t}\right\}$ with $\left(|G|, l_{i}\right)=1 \quad\left(l_{i} \in \mathbf{Z}, t \geq 2\right)$. In both cases, if $(G, \chi, 0)$ is sharp of type $L^{\sharp}$ as above, $G$ is either a sharply 3-transitive permutation group or a 2-transitive Forbenius group.
\end{abstract}

\section{INTRODUCTION}

M. Kiyota in his recent seminar talk [4] has introduced a class of finite groups with certain characters, called sharp triples, which class includes that of sharp pairs consisting of finite groups with sharp characters in the sense of Cameron and Kiyota. Where this theory of sharp pairs, which generalizes the notion of sharply multiply transitive permutation groups, has been developed and many examples are known $[2,5]$. We shall investigate the problem of determining sharp triples. In order to describe our results, we shall recall their definitions.

Let $G$ be a finite group with a character $\chi$ of degree $n, L$ the image of $\chi$ on $G$, and $L^{\sharp}=L \backslash\{n\}$. For each $l$ of $L, a(l)$ will denote the number of elements $x$ of $G$ with $\chi(x)=l$. Now the number $B(l)$ defined by $|a(l) /| G||$ $\prod_{k \in L \backslash\{l\}}(l-k)$ is an algebraic integer for each $l$ of $L$; this fact is known as a theorem of $\mathrm{H}$. F. Blichfeldt [1]. We shall call a triple $(G, \chi, l)$ a sharp

Received by the editors February 13, 1989 and, in revised form, December 18, 1989.

1980 Mathematics Subject Classification (1985 Revision). Primary 20C15; Secondary 20B20.

Key words and phrases. Characters of finite groups, Frobenius groups, transitive permutation groups. 
triple (or, simply, sharp) of type $L^{\sharp}$ provided that $B(l)$ is a unit in algebraic integers, and then the cardinality $\left|L^{\sharp}\right|$ is called the rank. In particular, when $\chi$ is faithful, a pair $(G, \chi)$ is called sharp of type $L^{\sharp}$ provided that $B(n)=1$, and then $\chi$ is also called sharp in the sense of Cameron-Kiyota. The problem above in the case of rank 2 was studied by the first author in [6]. This note will investigate it in special cases of rank $\geq 3$. Without loss of generality, we may assume that $\chi$ is a faithful character, so we always assume so throughout this article. Now we shall state our results.

Theorem 1. Let $G$ be a finite group with a faithful character $\chi$ of degree $n$, and let $L$ the image of $\chi$, and $L^{\sharp}=L \backslash\{n\}$. Assume that $L^{\sharp}$ consists of roots of unity in $\mathbf{C}$ together with $0 \in \mathbf{Z}$. If $(G, \chi, 0)$ is a sharp triple of type $L^{\sharp}$ of rank $\geq 2$, either (i) or (ii) below holds:

(i) $G$ is a sharply 3-transitive permutation group and $\chi$ is irreducible.

(ii) $G$ is a 2-transitive Frobenius group and $\chi$ is a sum of a linear character and the irreducible character induced from the kernel.

Remark. If $G$ is a sharply 3-transitive permutation group, then either $G \simeq$ $\operatorname{PGL}(2, q)$, or $G \simeq \operatorname{PSL}(2, q)\langle\alpha\rangle$ and $q$ is the square of an odd prime power, where $\alpha$ is the product of diagonal and field automorphisms of order 2 [7].

Furthermore, in the case $L^{\sharp} \subset \mathbf{Z}$ of rank $\geq 3$, we can prove the following:

Corollary 2. Let $G$ be a finite group with a faithful character $\chi$ of degree $n$, and let $L$ be the image of $\chi, L^{\sharp}=L \backslash\{n\}$. Let $m$ be any nonpositive integer and $l_{i}$ any nonzero integer for $i=1,2, \ldots, t$. Assume that each $l_{i}$ is prime to $|G|$. If $(G, \chi, m)$ is a sharp triple of type $L^{\sharp}=\left\{m, m+l_{1}, m+l_{2}, \ldots, m+l_{t}\right\}$ of rank $\geq 3$, either (i) or (ii) below holds:

(i) $G$ is a sharply 3-transitive permutation group and $\chi$ is irreducible; moreover, $\chi$ is sharp of type $\{-1,0,1\}$ in the sense of Cameron-Kiyota.

(ii) $G$ is a 2-transitive Frobenius group with an involutive linear character and $\chi$ is a sum of the linear character and the irreducible character induced from the kernel.

Our notation is largely standard. $A_{l}$ will denote the set $\{x \in G \mid \chi(x)=l\}$, and $a(l)$ the cardinality $\left|A_{l}\right|$ for $l$ of $L .1_{G}$ is the principal character of $G$.

\section{Proofs of TheOREM 1 AND COROLlary 2}

Before proceeding with the proofs of the theorem let us first note a congruence relation for character values:

Lemma. Let $G$ be a finite group with a character $\chi$. For any element $x$ of $G$, any integer $e$, and any prime $p$,

$$
\chi\left(x^{p^{e}}\right) \equiv \chi(x)^{p^{e}} \quad \bmod \mathfrak{p},
$$

where $\mathfrak{p}$ is a prime divisor of $p$. 
This immediately follows from the fact that $\chi(x)$ is a sum of $n$ roots of unity, where $n$ is the degree of $\chi$.

Proof of Theorem 1. Let $a=a(0)$, and $L^{\sharp}=\{0\} \cup\left\{\alpha_{1}, \alpha_{2}, \ldots, \alpha_{t}\right\}$, where each $\alpha_{i}$ is a root of unity. Set $k=\prod_{i=1}^{t} \alpha_{i}$, which is \pm 1 since $L$ is $\operatorname{Gal}(\bar{Q} / Q)$ invariant. By definition we have that $B(0)=(a /|G|) n k \in\{ \pm 1\}$ and so, from $B(n) \in N$, it follows that $|G|=$ an divides $n \prod_{i=1}^{t}\left(n-\alpha_{i}\right)$. First, let us note, $n \neq 1$; if not, then $G \subseteq A_{0}$, a contradiction. We also note that there exist distinct primes $p, q \in \pi(G)$ such that $p$ divides $n$ but $q$ does not divide $n$. This follows from $L^{\sharp} \backslash\{0\} \neq \varnothing$ by the lemma.

Now let us take any prime $p$ which divides $n$, and take any $x \in G$ of order $p$. We note $x \in A_{0}$ by the lemma. We shall claim that $\left|C_{G}(x)\right|=n$ and $A_{0}=$ $\operatorname{cl}_{G}(x)$ the conjugacy class of $x$ in $G$. Indeed, we shall first show that $\left|C_{G}(x)\right|$ divides $n$. For if it were not the case, as $\left|C_{G}(x)\right|$ divides $n \prod_{i=1}^{t}\left(n-\alpha_{i}\right)$, we can take $q \in \pi\left(C_{G}(x)\right)$, which does not divide $n$, and let $y \in C_{G}(x)$ of order $q$; then $z=x y$ is of order $p q$. The lemma gives $\chi\left(z^{q}\right)^{p} \equiv 0 \bmod \mathfrak{p}$, so $\chi\left(x^{q}\right)=\chi\left(z^{q}\right)=0$, and $\chi(x) \equiv \chi(z) \equiv 0 \bmod \mathfrak{q}$, where $\mathfrak{q}$ is a prime divisor of $q$, so we have $\chi(x)=\chi(z)=0$. However, since $\mathfrak{q}$ does not divide $n$, the same lemma also gives $\chi(z)^{p} \not \equiv 0 \bmod q$. We must have $\chi(z) \neq 0$, so $\chi(z)$ is a unit, contrary to the above, as asserted. Since $\operatorname{cl}_{G}(x) \subseteq A_{0}$, we have $a \leq\left(G: C_{G}(x)\right) \leq a$, so $\left|C_{G}(x)\right|=n$ and $\mathrm{cl}_{G}(x)=A_{0}$.

We note here, by the lemma, that $C_{G}(x)^{\sharp} \subseteq A_{0}=\mathrm{cl}_{G}(x)$. Thus, we have shown that $n$ is a power of $p$ and $C_{G}(x)$ is a $p$-subgroup.

We next claim that $C_{G}(x)$ is a Sylow $p$-subgroup of $G$ and $N_{G}\left(C_{G}(x)\right)$ is a 2-transitive Frobenius group. Indeed, $|G| / n \prod_{i=1}^{t}\left(n-\alpha_{i}\right)$; this yields, as $n$ and $\alpha_{i}$ are coprime, that $C_{G}(x)$ is a Hall subgroup. Thus we have shown that $C_{G}(x)$ is a Sylow $p$-subgroup of $G$ and $C_{G}(x)^{\sharp}$ is contained in the conjugacy class $\mathrm{cl}_{G}(x)$ for any element $x$ of $G$ of order $p$. Now we shall show that $P=C_{G}(x)$ is an elementary Abelian $p$-group; in fact, if $p=2$, this holds obviously. We may assume $p$ is odd. By Burnside's theorem [3, Theorem (7.1.1)], we have that $N_{G}(P)$ acts transitively on $Z(P)^{\sharp}$, so $N_{G}(P)$ has an involution. Since $C_{G}(x) \in \operatorname{Syl}_{p}(G)$ for any $x \in G$ of order $p$, the involution of $N_{G}(P)$ acts on $P$ fixed point freely, and hence, as $P^{\sharp} \subseteq \mathrm{cl}_{G}(x)$, we obtain that $P$ is elementary Abelian. Furthermore, by Burnside's theorem cited above, $N=N_{G}(P)$ acts transitively on $P^{\sharp}$, and $C_{N}(x)=P$ for any $x \in P^{\sharp}$, thus $N$ is a Frobenius group. Further, since $(N: P)=\left|P^{\sharp}\right|$ and $|N|=n(n-1)$, we have that $N_{G}\left(C_{G}(x)\right)$ is a 2-transitive Frobenius group, as asserted.

Let $b_{i}=a\left(\alpha_{i}\right) \quad(i=1,2, \ldots, t)$. Since $|G|=a n=1+a+\sum_{i=1}^{t} b_{i}$ and $\alpha_{i} \bar{\alpha}_{i}=1$, we have

$$
\langle\chi, \chi\rangle=\frac{1}{|G|}\left\{n^{2}+a n-(a+1)\right\} .
$$

Hence, since $C_{G}(x)^{\sharp} \subseteq A_{0}$, we have $n \leq a+1$, and thus, from $|G|=a n$, it 
follows that

$$
\langle\chi, \chi\rangle \leq 1+\frac{(n-1)}{a} \leq 2 .
$$

In the case where $\langle\chi, \chi\rangle=2$, we must have $a=n-1$, so $G=N_{G}\left(C_{G}(x)\right)$ is a 2-transitive Frobenius group with $\chi=\psi+\varphi^{G}$ (where $\psi$ is a linear character and $\varphi^{G}$ is the irreducible character induced from the kernel $\mathrm{P}$ ), which satisfies the hypothesis. In the case $\chi \in \operatorname{Irr}(G)$, from (1) and $|G|=a n$, it follows that $|G|=n\left(n^{2}-1\right)$. Hence, as $P=C_{G}(x)$ is a T.I. set, $G$ is a sharply 3-transitive permutation group on $\operatorname{Syl}_{p}(G)$. This completes the proof of Theorem 1 .

Proof of Corollary 2. Replacing $\chi$ by a character $\varphi=\chi-\mathrm{ml}_{G}$ we may as well assume that $(G, \varphi, 0)$ is sharp of type $L^{\sharp}=\left\{0, l_{1}, l_{2}, \ldots, l_{t}\right\}$. It is sufficient to show that $L^{\sharp}=\{-1,0,1\}$. Indeed, suppose this is the case, $G$ is a sharply 3-transitive permutation group or a 2-transitive Frobenius group by Theorem 1 . In the former case, $\varphi$ is a sharp character of type $\{-1,0,1\}$ in the sense of Cameron-Kiyota, since $|G|=n\left(n^{2}-1\right)$. In the latter case, as $L^{\sharp}$ is as above, $\varphi$ must be a sum of a nonprincipal involutive linear character and the irreducible character induced from the kernel. In both cases, since $\left\langle\varphi, 1_{G}\right\rangle=0$, we obtain $0 \leq\left\langle\chi, 1_{G}\right\rangle=m$, so $m=0, \chi=\varphi$; thus our reduction is valid.

Let $n$ be the degree of $\varphi$ and let $k=\prod_{i=1}^{t} l_{i} \in \mathbf{Z}$. As $(G, \varphi, 0)$ is sharp, it follows from the definition that $B(0)=(a /|G|) n k \in\{ \pm 1\}$, where $a=\mid\{x \in$ $G \mid \varphi(x)=0\} \mid$. So we have $|G|=$ anck, where $\varepsilon= \pm 1$ with $\varepsilon k>0$. Since each $l_{i}$ and $|G|$ are coprime, we obtain that $\varepsilon k=1$. Thus, as $\left|L^{\sharp}\right| \geq 3$, we have $t=2, l_{1}=-1$, and $l_{2}=1$, so $L^{\sharp}=\{-1,0,1\}$, as asserted. This completes the proof of Corollary 2.

\section{CONSEQUENCE AND REMARKS}

We shall call a character $\chi$ normalized provided $\left\langle\chi, 1_{G}\right\rangle=0$. As a consequence of Corollary 2 , we shall give a characterization of sharp triples of rank 3 with sharp characters, and shall state the result, without proof, as follows:

Let $G$ be a finite group with a faithful character $\chi$ of degree $n$, $L$ be the image of $\chi$, and $L^{\sharp}=L \backslash\{n\}$. Assume that $\chi$ is normalized. Then the following properties are equivalent:

(i) $(G, \chi, l)$ is a sharp triple of rank 3 with $L^{\sharp} \subset \mathbf{Z}$ for some $l \in L$, and $\chi$ is irreducible, sharp in the sense of Cameron-Kiyota.

(ii) $(G, \chi, 0)$ is a sharp triple of type $L^{\sharp}=\{-1,0,1\}$, and $\chi$ is irreducible.

(iii) $G$ is a sharply 3-transitive permutation group, and $\chi$ is irreducible, sharp of type $\{-1,0,1\}$ in the sense of Cameron-Kiyota.

As for Theorem 1(i), a sharply 3-transitive permutation group $G$ admits a sharp character $\chi=\varepsilon_{G}\left(\theta_{G}-1_{G}\right)$ of type $L^{\sharp}=\{-1,0,1\}$ in the sense of Cameron-Kiyota (where $\theta_{G}$ is the permutation character of $G$ and $\varepsilon_{G}$ is an 
involutive linear character of $G)$. Furthermore, $(G, \chi, 0)$ is also a sharp triple of type $L^{\sharp}$.

We note that a 2-transitive Frobenius group in Theorem 1 and Corollary 2 also admits the irreducible, sharp character of rank 2 , which is induced from the kernel.

As for Corollary 2 , in the case of rank 1 ; i.e. $(G, \chi, m)$ is sharp of type $\{m\}$, we have $G \simeq Z_{2}$ and $\chi=\varphi$ where $\varphi$ is the nonprincipal linear character of $Z_{2}[6]$.

\section{ACKNOWLEDGMENT}

The authors would like to express hearty thanks to Masao Kiyota for his kind comments on the first version of this article.

\section{REFERENCES}

1. H. F. Blichfeldt, $A$ theorem concerning the invariants of homogeneous groups with some applications in substitution groups, Trans. Amer. Math. Soc. 5 (1904), 461-466.

2. P. J. Cameron and M. Kiyota, Sharp characters of finite groups, J. Algebra 115 (1988), 125-143.

3. D. Gorenstein, Finite groups, Chelsea, New York, 1980.

4. M. Kiyota, Seminar talk at University of Tokyo, June 1987.

5. T. Matsuhisa, On finite groups admitting certain sharp characters, Bol. Soc. Bras. Mat. 18 (1987), 75-82.

6. _ A class of finite groups admitting certain sharp characters, Tsukuba J. Math. 14 (to appear).

7. H. Zassenhaus, Kennzeichunung endlicher linearer Gruppen als Permutationsgruppen, Abh. Math. Sem. Univ. Hamburg 11 (1936), 17-40.

Department of Mathematics, Ibaraki College of Technology, 866 Fukayatsu, KatSUTA, IBARAKI 312, JAPAN

Institute of Mathematics, University of Tsukuba, Ibaraki 305, Japan 